\title{
A MODIFIED VERSION OF TAYLOR'S HYPOTHESIS FOR SOLAR PROBE PLUS OBSERVATIONS
}

\author{
Kristopher G. Klein, Jean C. Perez, Daniel Verscharen, Alfred Mallet, and Benjamin D. G. Chandran \\ Space Science Center, University of New Hampshire, Durham, NH 03824, USA \\ Received 2014 December 11; accepted 2015 February 13; published 2015 March 4
}

\begin{abstract}
The Solar Probe Plus (SPP) spacecraft will explore the near-Sun environment, reaching heliocentric distances less than $10 R_{\odot}$. Near Earth, spacecraft measurements of fluctuating velocities and magnetic fields taken in the time domain are translated into information about the spatial structure of the solar wind via Taylor's "frozen turbulence" hypothesis. Near the perihelion of SPP, however, the solar-wind speed is comparable to the Alfvén speed, and Taylor's hypothesis in its usual form does not apply. In this paper, we show that under certain assumptions, a modified version of Taylor's hypothesis can be recovered in the near-Sun region. We consider only the transverse, non-compressive component of the fluctuations at length scales exceeding the proton gyroradius, and we describe these fluctuations using an approximate theoretical framework developed by Heinemann and Olbert. We show that fluctuations propagating away from the Sun in the plasma frame obey a relation analogous to Taylor's hypothesis when $V_{\mathrm{sc}, \perp} \gg z^{-}$and $z^{+} \gg z^{-}$, where $V_{\mathrm{sc}, \perp}$ is the component of the spacecraft velocity perpendicular to the mean magnetic field and $z^{+}\left(z^{-}\right)$is the Elsasser variable corresponding to transverse, non-compressive fluctuations propagating away from (toward) the Sun in the plasma frame. Observations and simulations suggest that, in the near-Sun solar wind, the above inequalities are satisfied and $z^{+}$fluctuations account for most of the fluctuation energy. The modified form of Taylor's hypothesis that we derive may thus make it possible to characterize the spatial structure of the energetically dominant component of the turbulence encountered by SPP.
\end{abstract}

Key words: plasmas - solar wind - Sun: corona - turbulence

\section{INTRODUCTION}

Understanding the processes that heat the Sun's corona and accelerate the solar wind is a long standing goal in the spacephysics community. A number of mechanisms have been proposed to account for these phenomena, including lowfrequency Alfvén-wave turbulence (Coleman 1968; Velli et al. 1989), cyclotron heating (Ionson 1978; Hollweg \& Isenberg 2002), stochastic heating (McChesney et al. 1987; Chaston et al. 2004; Chandran 2010), velocity filtration (Scudder 1992), reconnection (Parker 1972), and nanoflares (Parker 1988). Remote observations have been employed to constrain the likelihood of these mechanisms operating near the Sun (Hollweg et al. 1982; Harmon \& Coles 2005; De Pontieu et al. 2007; Tomczyk et al. 2007). However, in situ measurements are necessary to distinguish between these competing ideas.

The upcoming Solar Probe Plus (SPP) mission will make such measurements at heliocentric distances $r$ as small as $9.87 R_{\odot}$, where $R_{\odot}$ is the Solar radius. In order to extract scientific results from SPP measurements, we must consider the interpretation of measurements made in the spacecraft reference frame. Spacecraft measurements are made in the time domain, but are often translated into information about the spatial structure of waves and turbulence using Taylor's hypothesis (Taylor 1938; Fredricks \& Coroniti 1976), which treats fluctuations as static in the reference frame that moves with the mean velocity $\boldsymbol{U}$ of the solar wind (which we take to be measured in an inertial reference frame centered on the Sun). For example, if $\boldsymbol{B}_{\mathrm{sc}}(t)$ denotes the magnetic field measured by the spacecraft and $\boldsymbol{B}_{\mathrm{SW}}(\boldsymbol{x})$ denotes the (assumed-to-be) static magnetic field as a function of position $\boldsymbol{x}$ in the solar-wind frame, then

$$
\boldsymbol{B}_{\mathrm{sc}}(t)=\boldsymbol{B}_{\mathrm{SW}}\left(\boldsymbol{x}_{0}-\boldsymbol{U} t\right)
$$

where $\boldsymbol{x}_{0}$ is the spacecraft location at $t=0$. Here, we have neglected the velocity of the spacecraft, which, near Earth, is $\ll U$. Taylor's hypothesis is a good approximation when $U$ is much larger than the fluctuating plasma velocity and wave phase speeds, since waves and turbulent structures are then advected past the spacecraft on a timescale that is much shorter than the time required for the waves or structures to evolve appreciably in the solar-wind frame.

When Taylor's hypothesis holds and $\boldsymbol{U}$ is constant, the (angular) frequency power spectrum $P_{\omega}(\omega)$ of a quantity such as $\boldsymbol{B}_{\mathrm{sc}}(t)$ is related to the wavenumber spectrum $P_{3 \mathrm{D}}(\boldsymbol{k})$ of $\boldsymbol{B}_{\mathrm{SW}}(\boldsymbol{x})$ through the equation (Jokipii 1973; Horbury et al. 2008; Bourouaine \& Chandran 2013)

$$
P_{\omega}(\omega)=\int P_{3 \mathrm{D}}(\boldsymbol{k}) \delta(\boldsymbol{k} \cdot \boldsymbol{U}-\omega) d^{3} k,
$$

where the $k$ integration is over all of $k$-space. Thus, a wavenumber $\boldsymbol{k}$ in the solar-wind frame corresponds to an angular frequency $\omega=\boldsymbol{k} \cdot \boldsymbol{U}$ in the spacecraft frame.

A critical issue for SPP is that $U$ is comparable to the Alfvén speed $v_{\mathrm{A}}$ near SPP's perihelion, and thus Taylor's hypothesis does not in general apply. However, in this paper, we show that a modified version of Taylor's hypothesis can be recovered near SPP's perihelion under a set of conditions that are expected to hold for the energetically dominant component of the turbulent fluctuations in the near-Sun solar wind.

\section{A MODIFIED TAYLOR'S HYPOTHESIS FOR SPP}

In situ measurements indicate that the (total) fractional density fluctuations $\delta \rho / \rho_{0}$ in the solar wind at $r>60 R_{\odot}$ are much smaller than $|\delta \boldsymbol{B}| / B_{0}$, where $\delta \boldsymbol{B}$ and $\boldsymbol{B}_{0}$ are, respectively, the fluctuating and background magnetic fields, and $\delta \rho$ and $\rho_{0}$ are the fluctuating and background mass densities, respectively (Tu \& Marsch 1995). Observations of radio signals transmitted by the Helios spacecraft indicate that the inequality 
$\delta \rho / \rho_{0} \ll|\delta \boldsymbol{B}| / B_{0}$ also holds near SPP's perihelion (see, e.g., the appendix of Hollweg et al. 2010). The condition $\delta \rho / \rho_{0} \ll|\delta \boldsymbol{B}| / B_{0}$ implies that the dominant fluctuations are non-compressive, consistent with the fact that compressive waves are damped much more rapidly than non-compressive waves in the collisionless solar wind (Barnes 1966) and with Hollweg (1978)'s finding that fast magnetosonic waves launched outward through the chromosphere are reflected almost completely at the transition region.

In this paper, we restrict our analysis to the dominant, noncompressive component of the fluctuations and derive a version of Taylor's hypothesis that will apply only to such noncompressive fluctuations. We assume that

$$
\delta \rho \ll \rho_{0} .
$$

We also restrict our analysis to fluctuations at length scales greater than the proton gyroradius. At such scales, the only non-compressive mode is the Alfvén wave, for which $\delta \boldsymbol{B}$ and the fluctuating velocity $\delta \boldsymbol{v}$ are perpendicular to $\boldsymbol{B}_{0}$. We thus take the fluctuations to satisfy the conditions

$$
\nabla \cdot \delta \boldsymbol{v}=0 \quad \delta \boldsymbol{v} \cdot \boldsymbol{B}_{0}=0 \quad \delta \boldsymbol{B} \cdot \boldsymbol{B}_{0}=0 .
$$

We assume that $\boldsymbol{B}_{0}$ and the background solar-wind velocity $\boldsymbol{U}$ are, at least to a good approximation, in the radial direction $\hat{\boldsymbol{r}}$. (It is trivial to generalize our results to the case in which $\boldsymbol{B}_{0}$ points in the $-\hat{\boldsymbol{r}}$ direction.) We also take $\rho_{0}$ to vary with position perpendicular to $\hat{\boldsymbol{r}}$ much more slowly than does $\delta \boldsymbol{B}$. Given these assumptions and Equations (3) and (4), the fluctuations are described by the Heinemann \& Olbert (1980) equations (for additional discussion see Chandran \& Hollweg 2009),

$$
\begin{array}{r}
\frac{\partial \boldsymbol{g}}{\partial t}+\left(\boldsymbol{U}+\boldsymbol{v}_{\mathrm{A}}\right) \cdot \nabla \boldsymbol{g}-\left(\frac{U+v_{\mathrm{A}}}{2 v_{\mathrm{A}}}\right) \frac{d v_{\mathrm{A}}}{d r} \boldsymbol{f} \\
=-\boldsymbol{z}^{-} \cdot \nabla \boldsymbol{g}-\left(\frac{1+\eta^{1 / 2}}{\eta^{1 / 4}}\right) \frac{\nabla p_{\mathrm{tot}}}{\rho_{0}}
\end{array}
$$

and

$$
\begin{array}{r}
\frac{\partial \boldsymbol{f}}{\partial t}+\left(\boldsymbol{U}-\boldsymbol{v}_{\mathrm{A}}\right) \cdot \nabla \boldsymbol{f}-\left(\frac{U-v_{\mathrm{A}}}{2 v_{\mathrm{A}}}\right) \frac{d v_{\mathrm{A}}}{d r} \boldsymbol{g} \\
=-\boldsymbol{z}^{+} \cdot \nabla \boldsymbol{f}-\left(\frac{1-\eta^{1 / 2}}{\eta^{1 / 4}}\right) \frac{\nabla p_{\mathrm{tot}}}{\rho_{0}},
\end{array}
$$

where

$$
\boldsymbol{g}=\left(\frac{1+\eta^{1 / 2}}{\eta^{1 / 4}}\right) z^{+} \quad \boldsymbol{f}=\left(\frac{1-\eta^{1 / 2}}{\eta^{1 / 4}}\right) z^{-}
$$

are the Heinemann-Olbert variables, $\boldsymbol{v}_{\mathrm{A}}=\boldsymbol{B}_{0} / \sqrt{4 \pi \rho_{0}}$ is the Alfvén velocity, $p_{\text {tot }}$ is the total pressure,

$$
\eta \equiv \frac{\rho_{0}}{\rho_{a}}=\frac{v_{\mathrm{A}}^{2}}{U^{2}}
$$

$\rho_{a}$ is the value of $\rho_{0}$ at the Alfvén critical point $r=r_{\mathrm{a}}$ (at which $\left.U=v_{\mathrm{A}}\right)$,

$$
\boldsymbol{z}^{ \pm}=\delta \boldsymbol{v} \mp \delta \boldsymbol{b}
$$

are the Elsasser variables, and $\delta \boldsymbol{b}=\delta \boldsymbol{B} / \sqrt{4 \pi \rho_{0}}$. Physically, $\boldsymbol{g}$ and $z^{+}\left(f\right.$ and $\left.z^{-}\right)$represent noncompressive, transverse fluctuations that propagate away from (toward) the Sun in the plasma frame.

The pressure terms in Equations (5) and (6) enforce the incompressibility condition by canceling out the compressive components of the nonlinear terms $z^{+} \cdot \nabla \boldsymbol{f}$ and $z^{-} \cdot \nabla \boldsymbol{g}$. To simplify the notation, we define

$$
\left(z^{-} \cdot \nabla \boldsymbol{g}\right)_{\mathrm{nc}}=z^{-} \cdot \nabla \boldsymbol{g}+\left(\frac{1+\eta^{1 / 2}}{\eta^{1 / 4}}\right) \frac{\nabla p_{\mathrm{tot}}}{\rho_{0}},
$$

where the subscript "nc" stands for "non-compressive component."

Measurements from the Helios spacecraft show that on average the ratio $z^{+} / z^{-}$exceeds 1 at $0.3 \mathrm{AU}<r<1 \mathrm{AU}$ and that this ratio increases as $r$ decreases (Bavassano et al. 2000). Theoretical models (Cranmer \& van Ballegooijen 2005; Verdini \& Velli 2007; Chandran \& Hollweg 2009), shellmodel simulations (Verdini et al. 2012), and direct numerical simulations (Perez \& Chandran 2013) also suggest that $z^{+} \gg z^{-}$at $r \sim 10-30 R_{\odot}$. We thus assume that

$$
z^{-} \ll z^{+} \text {. }
$$

Equation (11) implies that $f \ll g$. Moreover, the ratio $f / g$ is much smaller than $z^{-} / z^{+}$near $r=r_{\mathrm{a}}$, because of the prefactor $\left(1-\eta^{1 / 2}\right) / \eta^{1 / 4}$ in the definition of $\boldsymbol{f}$ in Equation (7). The quantity $\left(1 / v_{\mathrm{A}}\right) d v_{\mathrm{A}} / d r$ is $\sim r^{-1}$. It thus follows from Equation (11) that $\left|\left(\boldsymbol{U}+\boldsymbol{v}_{\mathrm{A}}\right) \cdot \nabla \boldsymbol{g}\right| \gg \mid\left[\left(U+v_{\mathrm{A}}\right) /\right.$ $\left.\left(2 v_{\mathrm{A}}\right)\right]\left(d v_{\mathrm{A}} / d r\right) \boldsymbol{f} \mid$, because $|\partial \mathbf{g} / \partial r|$ is not much smaller than $|g / r|$. Given Equation (11), we may thus approximate Equation (5) as

$$
\frac{\partial \boldsymbol{g}}{\partial t}+\left(\boldsymbol{U}+\boldsymbol{v}_{\mathrm{A}}\right) \cdot \nabla \boldsymbol{g}=-\left(z^{-} \cdot \nabla \boldsymbol{g}\right)_{\mathrm{nc}} .
$$

We now change from a reference frame centered on the Sun with position $\boldsymbol{r}$ and time $t$ to the spacecraft reference frame with position $\boldsymbol{r}^{\prime}=\boldsymbol{r}-\int \boldsymbol{V}_{\mathrm{sc}} d t$ and time $t^{\prime}=t$. The temporal and spatial derivatives in the two frames are related by the equations $\partial / \partial t=\partial / \partial t^{\prime}+\partial \boldsymbol{r}^{\prime} / \partial t \cdot \nabla^{\prime}$ and $\nabla=\nabla^{\prime}$, where $\partial \boldsymbol{r}^{\prime} / \partial t=-\boldsymbol{V}_{\mathrm{sc}}$. We can thus rewrite Equation (12) as

$$
\frac{\partial \boldsymbol{g}}{\partial t^{\prime}}+\left(\boldsymbol{U}+\boldsymbol{v}_{\mathrm{A}}-\boldsymbol{V}_{\mathrm{sc}}\right) \cdot \nabla^{\prime} \boldsymbol{g}=-\left(z^{-} \cdot \nabla^{\prime} \boldsymbol{g}\right)_{\mathrm{nc}},
$$

where $\left(z^{-} \cdot \nabla^{\prime} g\right)_{\mathrm{nc}}$ is given by the right-hand side of Equation (10), with $\nabla$ replaced by $\nabla^{\prime}$.

Our goal now is to determine when the nonlinear term on the right-hand side of Equation (13) can be neglected. We note that the $z^{-} \cdot \nabla^{\prime} \boldsymbol{g}$ term "picks up" the spatial derivatives of $\boldsymbol{g}$ in the directions perpendicular to $\boldsymbol{B}_{0}$, because of Equation (4). On the other hand, the $\left(\boldsymbol{U}+\boldsymbol{v}_{\mathrm{A}}\right) \cdot \nabla^{\prime} \boldsymbol{g}$ term picks up the spatial derivatives of $\boldsymbol{g}$ along the direction of $\boldsymbol{B}_{0}$. Thus, even though $U+v_{\mathrm{A}} \gg z^{-}$, it is not necessarily the case that $\left|\left(\boldsymbol{U}+\boldsymbol{v}_{\mathrm{A}}\right) \cdot \nabla^{\prime} \boldsymbol{g}\right| \gg\left|z^{-} \cdot \nabla^{\prime} \boldsymbol{g}\right|$, because the perpendicular gradient of $\boldsymbol{g}$ could greatly exceed the parallel gradient. On the other hand, the spacecraft velocity $\boldsymbol{V}_{\mathrm{sc}}$ has a nonzero component $\boldsymbol{V}_{\mathrm{sc}, \perp} \equiv \boldsymbol{V}_{\mathrm{sc}}-\hat{b}\left(\hat{b} \cdot \boldsymbol{V}_{\mathrm{sc}}\right)$ perpendicular to $\boldsymbol{B}_{0}$. The term $\boldsymbol{V}_{\mathrm{sc}} \cdot \nabla^{\prime} \boldsymbol{g}$ thus greatly exceeds $\boldsymbol{z}^{-} \cdot \nabla^{\prime} \boldsymbol{g}$ in magnitude when

$$
V_{\mathrm{sc}, \perp} \gg z^{-} \text {. }
$$

Near perihelion, $V_{\mathrm{sc}, \perp} \simeq 200 \mathrm{~km} \mathrm{~s}^{-1}$ and Equation (14) likely holds (Cranmer \& van Ballegooijen 2005; Verdini \& 


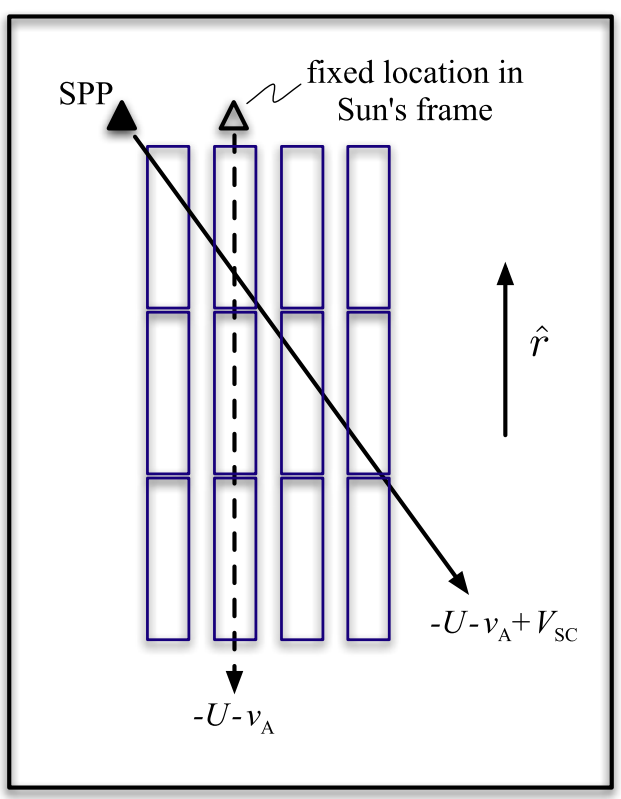

Figure 1. Motion of SPP (filled triangle) in the " $z$ r rest frame," which moves away from the Sun at velocity $\boldsymbol{U}+\boldsymbol{v}_{\mathrm{A}}$. The open triangle represents a point that is at rest in an inertial frame centered on the Sun. The rectangles represent turbulent structures associated with non-compressive $z^{+}$(or $g$ ) fluctuations.

Velli 2007; Chandran \& Hollweg 2009). When Equation (14) is satisfied, Equation (13) becomes (to leading order in the small quantity $\left.z^{-} / V_{\mathrm{sc}, \perp}\right)$

$$
\frac{\partial \boldsymbol{g}}{\partial t^{\prime}}+\boldsymbol{U}_{\mathrm{total}} \cdot \nabla^{\prime} \boldsymbol{g}=0
$$

where

$$
\boldsymbol{U}_{\text {total }} \equiv \boldsymbol{U}+\boldsymbol{v}_{\mathrm{A}}-\boldsymbol{V}_{\mathrm{sc}} .
$$

The left-hand side of Equation (15) is the convective time derivative of $\boldsymbol{g}$ at a point that moves with velocity $\boldsymbol{U}_{\text {total }}$. The vanishing of this time derivative to leading order in $z^{-} / V_{\mathrm{sc}, \perp}$ and $z^{-} / z^{+}$expresses mathematically the statement that $g$ fluctuations are approximately frozen in a frame that moves with velocity $\boldsymbol{U}_{\text {total }}$.

We now restrict our consideration to time intervals $\tau$ that are sufficiently short that (1) $\boldsymbol{V}_{\mathrm{sc}}$ is approximately constant and (2) $\boldsymbol{v}_{\mathrm{A}}$ and $\boldsymbol{U}$ are approximately constant at the spacecraft location and throughout the radial interval through which the $g$ fluctuations propagate during time $\tau$. With these conditions, $\boldsymbol{U}_{\text {total }}$ can be treated as constant, and the solution for $\boldsymbol{g}$ in Equation (15) is

$$
\boldsymbol{g}\left(\boldsymbol{r}^{\prime}, t^{\prime}\right)=\boldsymbol{g}\left(\boldsymbol{r}^{\prime}-\boldsymbol{U}_{\text {total }} t^{\prime}, 0\right) .
$$

Just as Equation (1) leads to Equation (2), Equation (17) implies that

$$
P_{\omega}^{(g)}(\omega)=\int P_{3 \mathrm{D}}^{(g)}(\boldsymbol{k}) \delta\left(\boldsymbol{k} \cdot \boldsymbol{U}_{\mathrm{total}}-\omega\right) d^{3} k,
$$

where $P_{\omega}^{(g)}$ is the (angular) frequency spectrum of $g$ in the spacecraft frame, and $P_{3 \mathrm{D}}^{(g)}$ is the wavenumber spectrum of $\boldsymbol{g}\left(\boldsymbol{r}^{\prime}, 0\right)$. In order for the inward-propagating fluctuations (i.e., $f)$ to obey an analogous relation, $\left|\boldsymbol{V}_{\mathrm{sc}, \perp}\right|$ would need to be $\gg \boldsymbol{z}^{+}$, which is not expected for the near-Sun environment.
To understand why a version of Taylor's hypothesis applies to outward-propagating $g$ fluctuations even when $U \sim v_{\mathrm{A}}$, it is helpful to view the spacecraft in the " $z$ reference frame," which moves away from the Sun at velocity $\boldsymbol{U}+\boldsymbol{v}_{\mathrm{A}}$, which, as discussed above, we treat as as effectively constant. In the $z^{+}$ reference frame, $\boldsymbol{g}$ changes only because of the nonlinear shearing represented by the $-\left(z^{-} \cdot \nabla \boldsymbol{g}\right)_{\mathrm{nc}}$ term on the righthand side of Equation (12). A fixed location in an inertial reference frame centered on the Sun moves at velocity $-\boldsymbol{U}-\boldsymbol{v}_{\mathrm{A}}$ in the $z^{+}$frame, as represented by the open triangle in Figure 1. At such a location, our modified version of Taylor's hypothesis (in this case with $V_{\mathrm{sc}}=0$ ) need not apply, because the timescale on which a $g$ structure evolves due to nonlinear shearing could be comparable to the time needed to traverse that structure in the radial direction at speed $U+v_{\mathrm{A}}$. This is directly related to the point made above that even though $U+v_{\mathrm{A}} \gg z^{-}$, it is not necessarily the case that $\left|\left(\boldsymbol{U}+\boldsymbol{v}_{\mathrm{A}}\right) \cdot \nabla^{\prime} \boldsymbol{g}\right| \gg\left|z^{-} \cdot \nabla^{\prime} \boldsymbol{g}\right|$, because $\boldsymbol{U}+\boldsymbol{v}_{\mathrm{A}}$ is along $\boldsymbol{B}_{0}$, whereas $z^{-}$is perpendicular to $\boldsymbol{B}_{0}$. On the other hand, in the $z^{+}$ frame, the SPP spacecraft will move at velocity $-\boldsymbol{U}-\boldsymbol{v}_{\mathrm{A}}+\boldsymbol{V}_{\mathrm{sc}}$, as illustrated by the filled triangle in Figure 1. If Equation (14) is satisfied, then SPP will traverse a $\boldsymbol{g}$ structure in a time that is much shorter than the time required for that structure to change in the $z^{+}$frame, and the $\boldsymbol{g}$ structures can be approximated as frozen.

Equations (17) and (18) are analogous to the usual forms of Taylor's hypothesis given in Equations (1) and (2), but with some differences. At $r=1 \mathrm{AU}$, structures are approximately frozen in the plasma frame and are swept by the spacecraft at a velocity $\simeq \boldsymbol{U}$. Closer to the Sun, when Equations (11) and (14) are satisfied, $\boldsymbol{g}$ structures are approximately frozen and are swept by the spacecraft at velocity $\boldsymbol{U}+\boldsymbol{v}_{\mathrm{A}}-\boldsymbol{V}_{\mathrm{sc}}$, but $\boldsymbol{f}$ structures need not be frozen. At $r=1 \mathrm{AU}$, the frequencies in the spacecraft frame are much larger than the frequencies of fluctuations measured in the plasma frame. In contrast, this frequency disparity need not arise near the Alfvén critical point. If the frequency associated with some fluctuation in the spacecraft frame arises primarily from the $\left(\boldsymbol{U}+\boldsymbol{v}_{\mathrm{A}}\right) \cdot \nabla^{\prime} \boldsymbol{g}$ term in Equation (13), then this frequency is only a factor of $\left(U+v_{\mathrm{A}}\right) / v_{\mathrm{A}} \sim 2$ larger than the frequency that would arise in the plasma frame.

The direct output of Equations (17) and (18) is the spatial structure of the $g$ field. However, because we restrict our analysis to time intervals $\tau$ that are sufficiently small that $U$ and $v_{\mathrm{A}}$ remain fairly constant at the spacecraft location and throughout a radial interval extending from SPP a distance $\sim\left(U+v_{\mathrm{A}}\right) \tau$ toward the Sun, the factor of proportionality between $z^{+}$and $g$,

$$
h \equiv \frac{\eta^{1 / 4}}{1+\eta^{1 / 2}},
$$

can be treated as approximately constant. As a consequence, the frequency and wavenumber spectra of $\boldsymbol{g}$ in Equation (18) can be converted into frequency and wavenumber spectra of $z^{+}$ via Equation (7). Near the Alfvén critical point $r_{\mathrm{a}}$, the radial variations in $h$ are particularly small, allowing for a translation between $\boldsymbol{g}$ spectra and $z^{+}$spectra with very little error. This can be seen by writing $\eta=1+x$, where $|x| \ll 1$ near $r=r_{\mathrm{a}}$. From Equation (8), $x=\left(v_{\mathrm{A}}^{2}-U^{2}\right) / U^{2}$. Taylor-expanding 
Equation (19) about $\eta=1$, we obtain

$$
h=\frac{1}{2}\left[1-\frac{\left(U^{2}-v_{\mathrm{A}}^{2}\right)^{2}}{32 U^{4}}+\ldots\right] .
$$

Because $h$ varies quadratically with the quantity $\left(U^{2}-v_{\mathrm{A}}^{2}\right)$, and because of the factor of $1 / 32, h$ varies very slowly with radius near $r_{\mathrm{a}}$.

To illustrate how the technique we describe could be applied, we suppose that near its perihelion of $9.87 R_{\odot}$ SPP traverses a fast-solar-wind stream emanating from a low-latitude coronal hole, in which $U$ and $v_{\mathrm{A}}$ are approximately steady for a period of $\tau=10^{3} \mathrm{~s}$, during which time $U+v_{\mathrm{A}}=1.3 \times 10^{3} \mathrm{~km} \mathrm{~s}^{-1}$ and $V_{\mathrm{sc}, \perp} \simeq V_{\mathrm{sc}}=2 \times 10^{2} \mathrm{~km} \mathrm{~s}^{-1}$. If Equations (11) and (14) are satisfied, then Equations (17) and (18) apply, and SPP measurements during this interval sample approximately frozen $g$ structures along a line segment that extends from $r=9.87 R_{\odot}$ to $r \simeq 9.87 R_{\odot}-\left(U+v_{\mathrm{A}}\right) \tau=7.99 R_{\odot}$, while spanning a distance $V_{\mathrm{sc}} \tau \simeq 0.29 R_{\odot}$ perpendicular to the radial direction. Over the radial range $\left(7.99 R_{\odot}, 9.87 R_{\odot}\right)$, the quantity $U+v_{\mathrm{A}}$ varies by $6.1 \%$ in the data-based model of Chandran \& Hollweg (2009), consistent with our assumption that $U+v_{\mathrm{A}}$ is reasonably constant throughout this radial interval. Given the density profile in this model, in which $r_{\mathrm{a}}=11.1 R_{\odot}$, Equations (8) and (19) imply that $h$ varies from a value of 0.491 at $r=7.99 R_{\odot}$ to a value of 0.499 at $r=9.87 R_{\odot}$. To within $2 \%, h=1 / 2$ throughout the interval $\left(7.99 R_{\odot}, 9.87 R_{\odot}\right)$, and thus the frequency and wavenumber spectra of $z^{+}$are to a high degree of accuracy equal to a constant (1/4) times the frequency and wavenumber spectra of g. As this example shows, the modified version of Taylor's hypothesis that we describe will enable SPP to probe the dominant component of solar-wind turbulence inside of SPP's perihelion.

In claiming that the energetically dominant fluctuations near the Sun approximately satisfy Equation (15), we have relied upon the assumptions that: (1) $\delta \rho / \rho_{0} \ll|\delta \boldsymbol{B}| / B_{0}$ (which in practice implies that $\delta \rho / \rho_{0} \ll 1$ since $\left.|\delta \boldsymbol{B}| \lesssim B_{0}\right)$; (2) $\delta \boldsymbol{B}$ and $\delta \boldsymbol{v}$ are approximately perpendicular to $\boldsymbol{B}_{0} ;(3) z^{+} \gg z^{-} ;$(4) $V_{\mathrm{sc}, \perp} \gg z^{-}$; (5) $\rho_{0}$ varies much more slowly with position perpendicular to $\hat{\boldsymbol{r}}$ than does $\delta \boldsymbol{B}$; and (6) $\boldsymbol{B}_{0}$ is nearly radial. SPP measurements will provide tests of conditions (1) through (5). To check assumption (6) using SPP measurements would require averaging $\boldsymbol{B}$ over timescales much longer than the timescale of the energetically dominant magnetic fluctuations, which is likely $\sim 1 \mathrm{hr}$ (Hollweg et al. 1982). However, such long time averages will become progressively more difficult as SPP approaches perihelion, since SPP will rapidly traverse the near-perihelion region at $\sim 1 R_{\odot} \mathrm{hr}^{-1}$. On the other hand, the assumption that $\boldsymbol{B}_{0}$ is nearly radial at $r \sim 10 R_{\odot}-30 R_{\odot}$ is likely accurate if coronal mass ejections are excluded, because the closed-loop magnetic structures of the Sun are primarily confined to smaller $r$ and the Parker spiral field begins to bend appreciably into the azimuthal direction only at larger $r$. We emphasize that the above conditions are sufficient but not necessary, since, e.g., Taylor's hypothesis can apply when $z^{+} \sim z^{-}$provided $z^{+} \ll V_{\mathrm{sc}, \perp}$. We also note that we have not restricted our analysis to fluctuations that are either quasi-2D or quasi-slab-symmetric, whether with respect to the background magnetic field $\boldsymbol{B}_{0}$ or the local magnetic field $\boldsymbol{B}_{0}+\delta \boldsymbol{B}$.
Observations at $1 \mathrm{AU}($ Podesta \& Bhattacharjee 2010) and numerical simulations of solar-wind turbulence near the Sun (Verdini et al. 2012; Perez \& Chandran 2013) suggest that if the overall fractional cross helicity is high, then $z^{+} \gg z^{-}$ throughout much of the inertial range. If this is the case near the Sun, then $\delta \boldsymbol{b} \simeq(1 / 2) \delta \boldsymbol{z}^{+}$scale by scale throughout much of the inertial range, and SPP magnetometer measurements on their own (without velocity measurements) will be sufficient to approximate the frequency spectra of $g$ and $z^{+}$.

Our study differs from previous studies of Taylor's hypothesis for SPP. Matthaeus (1997) accounted for $V_{\mathrm{sc}, \perp}$ and concluded that the characteristic turbulence speed must be $\ll V_{\text {sc, } \perp}$ for Taylor's hypothesis to apply to 2D turbulence. In contrast, we have shown that a form of Taylor's hypothesis applies to $\boldsymbol{g}$ or $\boldsymbol{z}^{+}$fluctuations even when the characteristic turbulence speed is comparable to $V_{\mathrm{sc}, \perp}$, provided $z^{-} \ll z^{+}$and

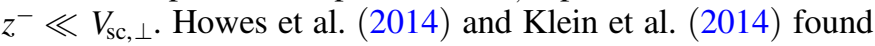
that Taylor's hypothesis holds near the perihelion of SPP for sufficiently oblique Alfvén and kinetic Alfvén waves if the fluctuations are treated as linear waves. In the present study, we do not restrict the analysis to linear or highly oblique waves.

\section{SUMMARY AND CONCLUSION}

Transverse, non-compressive fluctuations likely comprise the bulk of the fluctuation energy in the near-Sun solar wind. When $\delta \rho \ll \rho_{0}, \boldsymbol{U}$ and $\boldsymbol{B}_{0}$ are nearly radial, and $\rho_{0}$ varies much more slowly with position perpendicular to $\hat{\boldsymbol{r}}$ than does $\delta \boldsymbol{B}$, these transverse, non-compressive fluctuations are described by the Heinemann-Olbert equations. We use these equations to show that outward-propagating fluctuations can be treated as frozen within a reference frame that will be advected past SPP at velocity $\boldsymbol{U}+\boldsymbol{v}_{\mathrm{A}}-\boldsymbol{V}_{\mathrm{sc}}$, provided that $z^{-} \ll z^{+}$and $z^{-} \ll V_{\mathrm{sc}, \perp}$. Observations and simulations suggest that these latter two inequalities and the inequality $\delta \rho \ll \rho_{0}$ will be satisfied near SPP's perihelion. As a consequence, it will likely be possible to use a modified version of Taylor's hypothesis to characterize the spatial structure of the outward-propagating, transverse, non-compressive fluctuations that will be encountered by SPP near its perihelion, even when $U \sim v_{\mathrm{A}}$ and $z^{+} \sim V_{\mathrm{sc}}$.

This work was supported in part by NSF grants AGS1331355 and AGS-1258998, grant NNX11AJ37G from NASA's Heliophysics Theory Program, NASA grant NNN06AA01C to the Solar Probe Plus FIELDS Experiment, and NASA grant NNX12AB27G.

\section{REFERENCES}

Barnes, A. 1966, PhFl, 9, 1483

Bavassano, B., Pietropaolo, E., \& Bruno, R. 2000, JGR, 105, 15959

Bourouaine, S., \& Chandran, B. D. G. 2013, ApJ, 774, 96

Chandran, B. D. G. 2010, ApJ, 720, 548

Chandran, B. D. G., \& Hollweg, J. V. 2009, ApJ, 707, 1659

Chaston, C. C., Bonnell, J. W., Carlson, C. W., et al. 2004, JGRA, 109, 4205

Coleman, P. J., Jr. 1968, ApJ, 153, 371

Cranmer, S. R., \& van Ballegooijen, A. A. 2005, ApJS, 156, 265

De Pontieu, B., McIntosh, S. W., Carlsson, M., et al. 2007, Sci, 318, 1574

Fredricks, R. W., \& Coroniti, F. V. 1976, JGR, 81, 5591

Harmon, J. K., \& Coles, W. A. 2005, JGR, 110, 3101

Heinemann, M., \& Olbert, S. 1980, JGR, 85, 1311

Hollweg, J. V. 1978, GeoRL, 5, 731

Hollweg, J. V., Bird, M. K., Volland, H., et al. 1982, JGR, 87, 1

Hollweg, J. V., Cranmer, S. R., \& Chandran, B. D. G. 2010, ApJ, 722, 1495

Hollweg, J. V., \& Isenberg, P. A. 2002, JGRA, 107, 1147

Horbury, T. S., Forman, M., \& Oughton, S. 2008, PhRvL, 101, 175005 
Howes, G. G., Klein, K. G., \& TenBarge, J. M. 2014, ApJ, 789, 106 Ionson, J. A. 1978, ApJ, 226, 650

Jokipii, J. R. 1973, ARA\&A, 11, 1

Klein, K. G., Howes, G. G., \& TenBarge, J. M. 2014, ApJL, 790, L20

Matthaeus, W. H. 1997, Robotic Exploration Close to the Sun: Scientific Basis, Vol. 385 (New York: AIP), 67

McChesney, J. M., Stern, R. A., \& Bellan, P. M. 1987, PhRvL, 59, 1436

Parker, E. N. 1972, ApJ, 174, 499

Parker, E. N. 1988, ApJ, 330, 474
Perez, J. C., \& Chandran, B. D. G. 2013, ApJ, 776, 124

Podesta, J. J., \& Bhattacharjee, A. 2010, ApJ, 718, 1151

Scudder, J. D. 1992, ApJ, 398, 299

Taylor, G. I. 1938, RSPSA, 164, 476

Tomczyk, S., McIntosh, S. W., Keil, S. L., et al. 2007, Sci, 317, 1192

Tu, C., \& Marsch, E. 1995, SSRv, 73, 1

Velli, M., Grappin, R., \& Mangeney, A. 1989, PhRvL, 63, 1807

Verdini, A., Grappin, R., Pinto, R., \& Velli, M. 2012, ApJL, 750, L33

Verdini, A., \& Velli, M. 2007, ApJ, 662, 669 